\title{
Time Line Correlative Spectral Processing for Stratification of Blood Pressure using Adaptive Signal Conditioning
}

\author{
Santosh Shinde ${ }^{1}$ \\ Research Scholar, Department of Computer Science and \\ Engineering, Koneru Lakshmaiah Education Foundation \\ Vaddeswaram, AP, India-522502
}

\author{
Pothuraju RajaRajeswari ${ }^{2}$ \\ Professor, Department of Computer Science and Engineering \\ Koneru Lakshmaiah Education Foundation \\ Vaddeswaram, AP, India-522502
}

\begin{abstract}
Stratification of Blood Pressure is essential input in most of the cardiovascular diseases detection and prediction and is also a great aid to medical practitioners in dealing with Hypertension. Denoising based on spectral coding is developed based on frequency spectral decomposition and a spectral correlative approach based on wavelet transform. The existing approaches perform a standard deviation and mean of peak correlation in signal conditioning. The artifact filtrations were developed based on thresholding. Filtration of coefficients has an impact on accuracy of estimation and hence proper signal conditioning is a primal need. Wherein threshold is measured with discrete monitoring, time line observation could improve the accuracy of filtration efficiency under varying interference condition. Dynamic interference due to capturing or processing source results in jitter type noises which are short period deviations with varying frequency component. Hence a timefrequency analysis for filtration is adapted for filtration. This paper presents an approach of spectral correlation approach for signal condition in stratification of blood pressure under cuff less monitoring. This presented approach operates on the spectral distribution of finer resolution bands for monitoring signal in denoising and decision making. Existing approaches lacks the capability of loss-less denoising which is efficiently worked out in this paper.
\end{abstract}

Keywords-Stratification of blood pressure; discrete wavelet transform; spectral coding; and selective correlative approach

\section{INTRODUCTION}

Monitoring of blood pressure is a vital monitoring for automated diagnosis in cardio vascular disease diagnosis. A deflected blood pressure (BP) has a high risk of heart disorderness or heart failure. An early detection and diagnosis is an optimal solution to the asserted risk. The present diagnosis is a cuff based monitoring where the device is interfaced to patient hand to read the mercury level for BP monitoring. The interfacing of such devices is limited to expert personals and not users friendly in handling. With advancement of technology new approaches has evolved in diagnosis, wherein BP monitoring is also improved towards cuff-less measurement. Pulse transient time (PTT) [1-4] is a majorly observed approach in this type of measurement. The analysis presents a good correlation in diagnosis of PTT with BP monitoring. In [5] author interfaced electrocardiograph (ECG) with photoplethysmography (PPG) signals to estimate the BP level. A correlative approach in measuring mean error (ME) for the measured BP and the actual measurement is evaluated based on standard deviation for the magnitude values. In [6] boosting approach is presented in deriving a more calibrated monitoring of BP using ME. The impact of monitoring is however limited in such approach with the interference of external distortion.

This paper presents a novel Time line correlative spectral processing approach for efficient denoising of Vital BioSignals such as PPG, ECG and ABP. It utilizes an adaptive signal conditioning approach. The proposed method results in minimum loss in terms of Frequency components after wavelet computations and hence preserves the significant medical information in the signal.

To present the outlined approach this paper is outlined into seven sections. Section 2 presents the Background work citing the challenges and limitations of existing approaches. Section 3 outlined the existing approach of signal processing and analysis. Section 4 presents the approach of denoising signal in a nonlinear distortion approach. Section 5 outlines the simulation result for the developed approach. Section 6 presents Discussion and Future Work. The conclusion for the developed approach is presented in Section 7. Section 8 is about acknowledgment.

\section{BACKGROUND}

The filtration of the distorted signal in BP monitoring were developed in past where a threshold based estimation approach is proposed in [4] for PLI noise elimination. The approach developed a correlative method in minimizing the distortion using spectral energy variation among the normal and effective signal. In [7,8] a wavelet based estimation is proposed based on threshold approach where the signal is processed based on wavelet coefficients and measured threshold value. The correlation performs an elimination of the distortion effect based compared threshold. However it has an elimination of required signal coefficient as well. To eliminate the effect a KNN based approach was presented in [9]. Varying window of filtration for signal processing is outlined in [10]. The Hilbert transformation approach is developed in signal processing for varying interference. Feature detection based on normalized multi wavelet measure is outlined in [11]. The approach developed evaluate based on Euclidian distance. A simple 
segmentation approach for signal processing is outlined in [12], where multiple features based on spectral representation is outlined. The approach works on the geographical feature is presented. A time based estimation where the time parameter of magnitude is outlined in $[13,14]$. This approach is developed for a temporal sequence where time based features are used in deriving the observations for the determination of the peak parameter as feature values. The denoising based on DWT is developed based on frequency spectral decomposition and the range of frequency value is considered as the noised element wherein a standard deviation and mean of peak is considered for denoising. However, the decision is developed based on threshold value. The elimination of coefficient has an impact on accuracy of estimation hence threshold valuation is a critical issue. Wherein threshold is measured with discrete monitoring, time line observation could improve the accuracy of threshold detection and denoising efficiency. It is observed that sampling of signal for processing result in jitter [15-18] which are short period variation with different frequency component, which limits the DWT based denoising approach. The assumption of frequency range elimination for denoising leads to information loss and appropriate selection of coefficient could improve the accuracy. We propose to develop a new denoising approach to signal processing by developing time line processing and analysis for threshold computation. Secondly, we propose to integrate the DWT based approach with standard deviation and mean of peak over decomposed Time line (TL) to improve denoising performance. Towards developing a filtration of processing signal for distortion minimization in this work a time line approach for signal denoising using spectral decomposition and spectral correlation is outlined.

Machine Learning and Deep Learning is becoming increasingly popular in health informatics [21], [23-24], [2729] [31]. Further Compute-Intensive Large Scale Servers and Cloud Storage could optimize the effectiveness in terms of storage and transmission which will be secure for such data systems in future development efforts [19], [20], [22], [25].

\section{Photoplethysmography (PPG) SignAl ANALYSIS FOR BP STRATIFICATION}

Blood pressure is an important physiological parameter in the human body and it is critical indicator of clinical condition in both emergency situations and daily health care basis. In modern society of rich living, high blood pressure is constantly increasing. At the same time, people are more cautious about their health. Blood pressure (BP) is a force in the bloodstream flowing in the blood vessels and chambers of the heart. It is measured as the amount of blood flow in the arterial arteries, which is the main blood vessel that transmits blood from the heart. BP of a person is usually measured as a systolic pressure due to contraction cycle of the heart and as a diastolic pressure during its relaxation state. Average values of healthy people at systole read $80 \mathrm{~mm}$ of $\mathrm{Hg}$ and $120 \mathrm{~mm}$ of $\mathrm{Hg}$ diastole at stable condition [1]. Some factors that affect a person's blood pressure are pumping rates, hypertension, immune suppression, and blocks in respiratory airways. Because of various reasons, the average blood pressure is different from each individual. Blood pressure is categorized into five main scope-based on measured values as shown in Table I.
TABLE I. CATEGORIES OF BLOOD PRESSURE [1]

\begin{tabular}{|l|l|l|}
\hline Category & Systolic $(\mathbf{m m H g})$ & Diastolic $(\mathbf{m m H G})$ \\
\hline Hypotension & $<90$ & $<60$ \\
\hline Normal & $90-120$ & $60-80$ \\
\hline Prehypertension & $121-139$ & Or $81-89$ \\
\hline Stage 1 Hypertension & $140-159$ & Or $90-99$ \\
\hline Stage 2 Hypertension & $>160$ & Or $=100$ \\
\hline
\end{tabular}

According to the Seventh Report of the Joint National Committee for Prevention, Detection, Evaluation, and High Blood Pressure, hypertension is an elevated blood pressure, which leads to vascular damages of internal organs. It is considered to be a critical issue, as most of the patients do not realize the symptoms of the elevated blood pressure. Hypotension refers to a state of low blood pressure due to inadequate supply of blood to other organs in the body. The drop in blood pressure consequently results in heart attacks, strokes, kidney failure and other more serious complications. The monitoring of cuff less blood pressure is hence a prime need in current health monitoring approach. Blood pressures are monitored via different bio-signals such as ECG, PPG, etc. In our previous work we have developed a Hybrid framework for BP estimation utilizing both ECG and PPG signals [30]. However, the approach of representative features and the quality of signal plays a vital role in making decision. The analysis of PPG is observed to be more effective in BP monitoring due to confined feature representation. In processing of PPG signal, the electrical distortions are the major concern. The distortion has a greater impact in the accuracy of decision and hence need to be removed to the finest level. In PPG signal processing, the $50-60 \mathrm{~Hz}$ power line noise are one major source of distortion. This noise is observed stationary and majorly observed in the signal processing. The baseline distortion is considered to be due to respiration artifact is observed to be of low-frequency and the component is effective in time domain due to saturation of analog component in the processing system. The high frequency components are observed to be due to muscular movement in the body. Filtration of such artifacts is of primal need in processing of bio-signal to highest accuracy. Wavelet based spectra coding were observed in recent past for the filtration of noise component and need a finer processing for filtration. The approach of DWT based processing decompose the signal into finer TLs where a low range frequency of $0-0.25 \mathrm{~Hz}$ referring to baseline and a higher frequency range of $20-500 \mathrm{~Hz}$ reflect to power line harmonic and muscular based artifact where zero coefficient are removed. The decomposed coefficient is processed to denoising based on threshold approach. The deviation is measured based on mean peak value used for denoising. The representation of PPG signal is illustrated in "Fig. 1".

The process of Bio-signal processing for BP Stratification is developed in three steps of operations:

1) Preprocessing.

2) Feature extraction.

3) Learning and Decision system. 


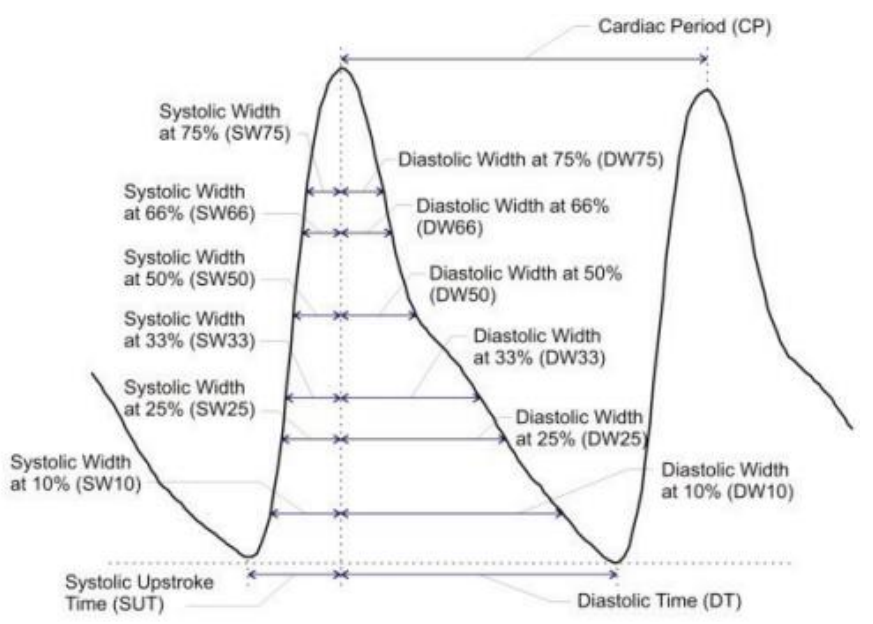

Fig. 1. PPG Signal Representation [3].

Preprocessing is developed for elimination of noise and artifacts in signal captured. In recent, DWT based approach was presented with a specified low and high frequency range for noise suppression. A standard deviation and mean peak based denoising is presented in [1]. Denoising is developed based on threshold limits. The limiting threshold is developed based on discrete magnitude monitoring, which is limited for usage for randomly varying time variant distortions. The sampling process adds jitter noise to the processing signal. The issue of discrete monitoring is focused and a new estimation approach based on time line observation is proposed. This approach develops a denoising threshold based on set of coefficients for a time interval. The process of denoising based on fixed cutoff approach is proposed to improve by a time line monitoring of standard deviation and peak (valley) for decomposed sub frequency bands. The noise parameter is developed for jitter analysis and harmonic content are observed in the processing signal. The decomposed signal is processed with a varying of frequency component is directly extracted and correlated using distortion minimization using spectral coding. The signal analysis is developed for the selection of a spectral power density using correlation approach. The derivative component of the filtration approach is processed for noise reduction in consider to lower magnitude value based on the deviation of the distortion in the signal processing. The processing of signal is divided into decomposed bands which eliminate the basic frequency for the process of no DC component. The process is developed based on the decomposition of processing signal into finer frequency bands and performs an extreme spectral selection. The process of decomposition for a given processing signal $\mathrm{S}(\mathrm{t})$ is represented by the spectral resolution $(\varphi)$ and the residual component res[i] is presented by,

$S[t]=\sum_{i=1}^{K} \varphi_{j}[i]+\operatorname{res}[i]$

The processing bio-signal is recorded as a set of 3 channels data, where the 1st channel represents the PPG signal, 2nd signal represents the arterial blood pressure (ABP), and the 3rd channel represented the ECG signal of monitoring signal. Wherein channel 1 and 3 are the monitoring signal, channel 2 is taken as the baseline representation of the BP signal. In evaluation of the denoising process in this work, 2 nd channel signal (ABP) is processed with varying noise levels to evaluate the efficiency of proposed approach. The baseline reference of ABP presents the level of analysis with the noised signal. The proposed approach of spectral correlation filtration approach for the Bio-signal in BP stratification is presented in next section. More noisy situations restricts the real time use of many such and similar systems [26].

\section{Selective Time Line Spectral Correlative PROCESSING (TLSC)}

This proposed approach focus in improving the denoising performance by minimizing the interference level and improving the details of representation. The spectral band extraction in time-frequency domain is proposed to represent physiological parameters and spectral parameters for denoising signal. A feature representation and classification process is used as an integral module for learning and decision making. The proposed processing system is for signal conditioning is illustrated in "Fig. 2".

\section{A. Spectral Decomposition Coding}

The signal analysis of the processing signal is performed based on the spectral decomposition and correlation process where the following two process were performed,

1) Extreme bands decomposition is developed with the successive decomposition of signal using cascaded filters.

2) Selecting local minima parameter for noised component based on local maxima of spectral density in spectral bands.

The process of filtration is developed with the normalization of processing signal based on mean parameter given as,

$\mathrm{m}_{1}=\mathrm{S}(\mathrm{t})-\mathrm{mn}_{1}$

The mean normalized signal set the processing signal to mean level, and random glitches are truncated to a mean reference. This normalized signal is then processed for spectral decomposition in denoising operation. The process of denoising is performed using a cascaded filter bank as shown in "Fig. 3".

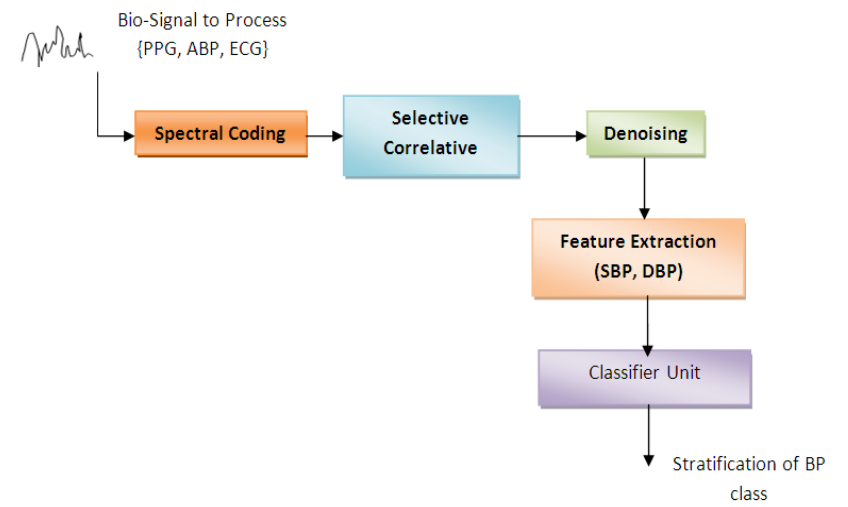

Fig. 2. Proposed Systems for Signal Analysis. 


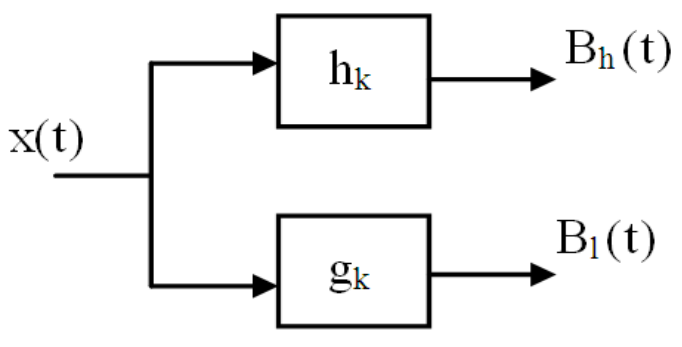

Fig. 3. Decomposition Filter Bank Structure.

The processing signal is represented as in "Fig. 4",

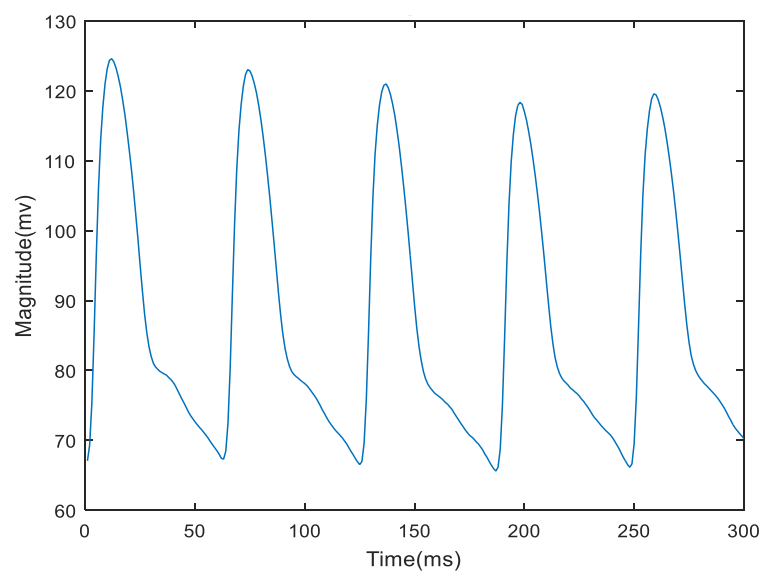

Fig. 4. An ABP Signal Plot for Processing.

The monitoring input to the Decision system is given by the spectral feature developed by band decomposition. A successive integration of filter banks results in a decomposition of signal into finer frequency band. For the realization of such filtration a wavelet based filtration is used. In the process of spectral decomposition approach, successive filter bank architecture is used as shown in "Fig. 5".

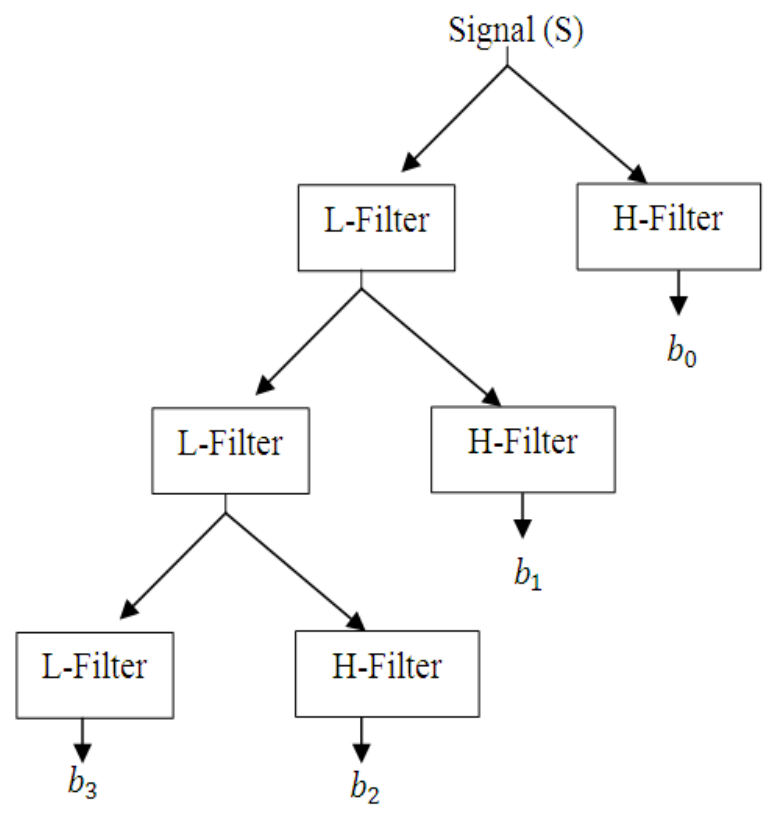

Fig. 5. Hierarchical Filtration Structure for Band Decomposition.

\section{B. Spectral Correlative Denoising Approach}

For spectral denoising each of the signal (S) is processed for B-Bands decomposition which represent a set of decomposed bands $\left\{b_{0}, b_{1}, b_{3} \ldots \ldots b_{n}\right\}$ given as,

$B=\left\{b_{0}, b_{1}, b_{3} \ldots \ldots b_{n}\right\} \in S$

The filtration of the noise component is developed be the convolution operation of given signal with the selected filtered signal $\mathrm{F}$,

$\mathrm{B}=\operatorname{conv}(\mathrm{S}, \mathrm{F})$

The High pass (HP) and low pass (LP) filter are developed as FIR filter giving the spectral variation of the observing parameter. Information's lower than the threshold is neglected. In this approach by the acquisition of vital parameter coding and normalization process is proposed. The acquisition process converts the vital parameter information into a 1-D plane. The resultant information is then processed for extracting the features based on Wavelet coding. This suggested method improves the selection of feature relevancy, in terms of selectivity. Since, now features are selected based on variation density rather than magnitudes. For the acquisition of the spectral coding curve, a linear sum of the entire vital parameter plane at different Gaussian smoothening factor is taken.

Each decompose band mean spectral density (MSD) is computed given by,

$M S D=\frac{\sum_{i=1}^{n}\left|B^{2}\right|}{n}$

$\mathrm{B}$ is the decomposed band for a signal $\mathrm{S}$ which reflect the measured parameter for a period ' $t$ '.

For each of the band decomposed is given by,

$\operatorname{MSD}(i)$, for $i=1$ to $n$.

Where $\mathrm{n}$ defines the number of decompositions.

For derived MSDi, the maximum MSD is given as,

$\mathrm{MxPi}=\max (\mathrm{MSDi})$

For $\mathrm{i}=1$ to $\mathrm{n}$

$$
\begin{gathered}
\text { if }(\mathrm{MSDi} \geq(\mathrm{MxPi} / 2)) \\
\text { sel_Bi }=\mathrm{Bi},
\end{gathered}
$$

End

The selected SBSs, 'sel_Bi' are only considered in processing where other bands are suppressed to zero. An inverse filtration operation with selected band and other band valued to zero is performed using convolution and successive addition process. This process results in de-noised signal with maximum information's suppressing noise distortion.

\section{Simulation Result}

For the Stratification of Blood Pressure using signal processing, a correlative spectral approach is presented. Wherein the existing approach operates on discrete coordinates and a correlation with the threshold is used in decision, the discrete value can be biased due to miss-operation of sensor, or 
measuring devices which effect the decision. Hence, a time line continuous monitoring in time-frequency domain result in more decision accuracy compared to discrete monitoring. It is observed that each of the observation has a systolic, Diastolic parameter which is time variant. In the developed approach the processing signal ABP is extracted from the 3 recorded channel and channel 2 is used for the noised component filtration analysis. The processing signal with Gaussian distortion have variance $($ var $)=0.01$ is shown in "Fig. 6".

The noised signal is represented to decomposed sub band which is carried out using ' $\mathrm{db} 4$ ' wavelet decomposition to compute the spectral band (SBSs). The 5 sub bands extracted from the processing signal is shown in "Fig. 7".

The MSD parameter for 4 detail SBSs and 1 residual SBSs is illustrate in "Fig. 8". The decomposed bands are processed for selection based on the magnitudes of these bands.

The representation of correlation of spectral bands with the decomposed band is illustrated in "Fig. 9". The band with a maximum MSD is selected with other band suppressed to zero.

The inverse process of selected band with other zero suppressed in inverse filtered and successive added to produce de-noised signal. Obtained de-noised signal is shown in "Fig. 10".

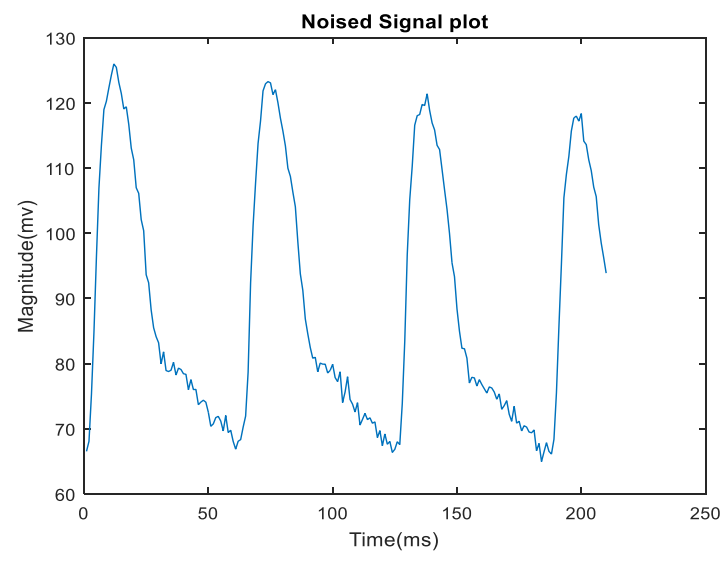

Fig. 6. Noised Signal with Variance (var=0.01).

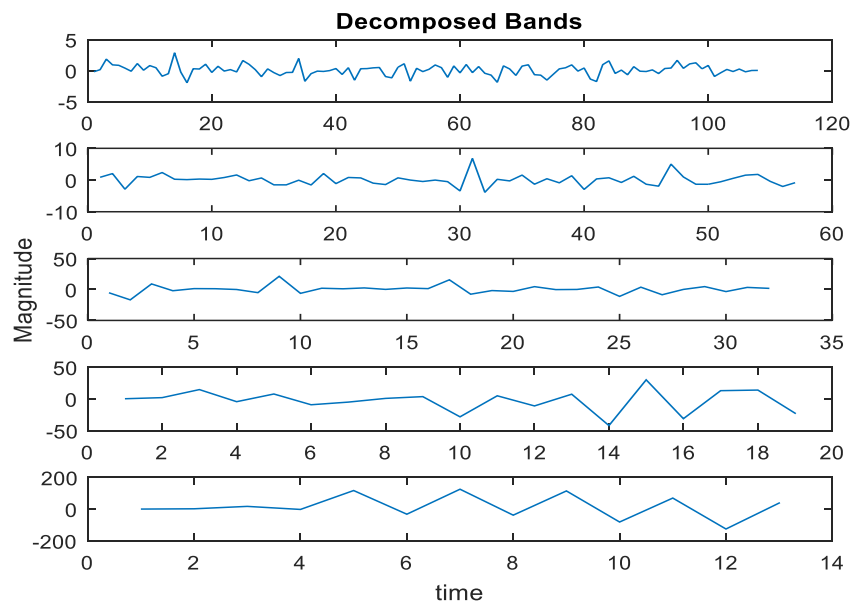

Fig. 7. SBSs Extracted from Processing Signal for Analysis.

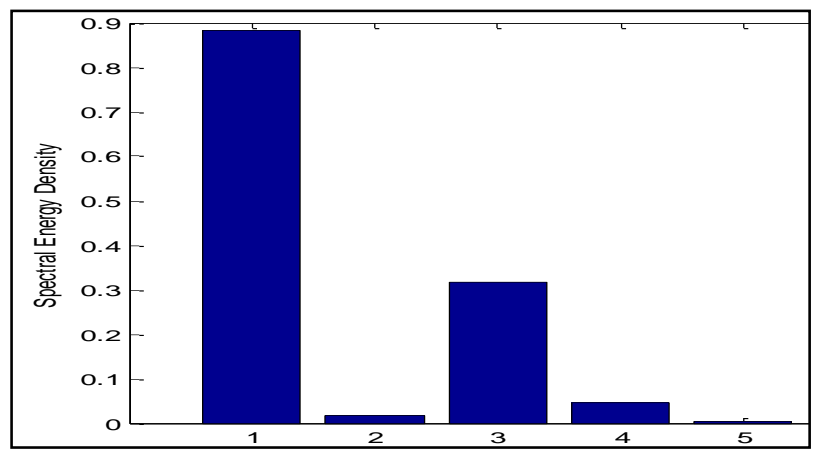

Fig. 8. MSD for the Spectral Bands Decomposed.

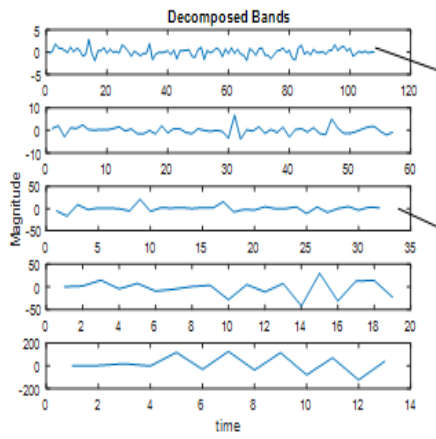

(a)

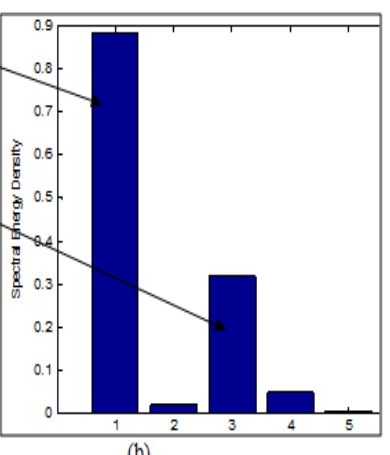

(b)
Fig. 9. (a) SBS for the Signal (b) MSD Correlation.

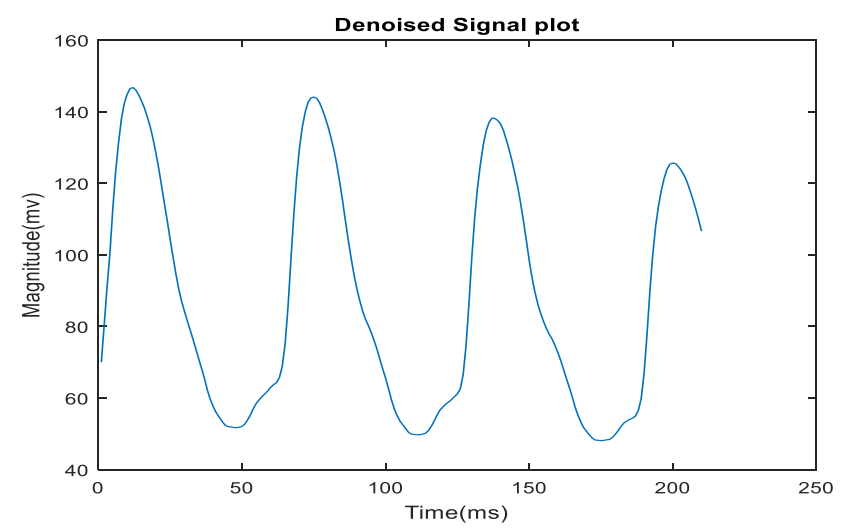

Fig. 10. De-noised Signal.

The de-noised signal is processed for feature extraction where the two extremes SBP and DBP is measured. The detected SBP and DBP detected for the de-noised signal is shown in "Fig. 11".

The decision system process on the computed DBP and SBP for a period of observation and an average majority of the BP stratification as derived from the condition listed in Table I is made. The decision system generates an alert for the decision made as shown in "Fig. 12".

A test of 10 iterations with varying Variance value from 0 to 10 is performed. The decision is monitored and an average accuracy is computed. The denoising efficiency is measured by an average Means square error (MSE), Peak signal to noise ratio (PSNR) and Root mean square error (RMSE) values. 


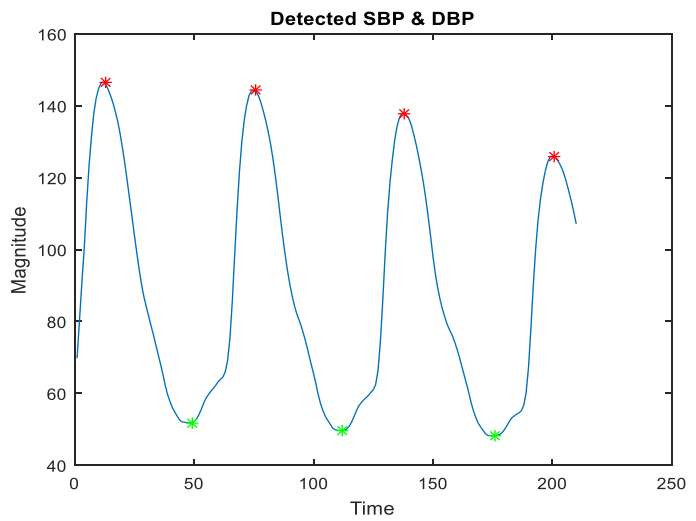

Fig. 11. Detected Peak Points for Decision.

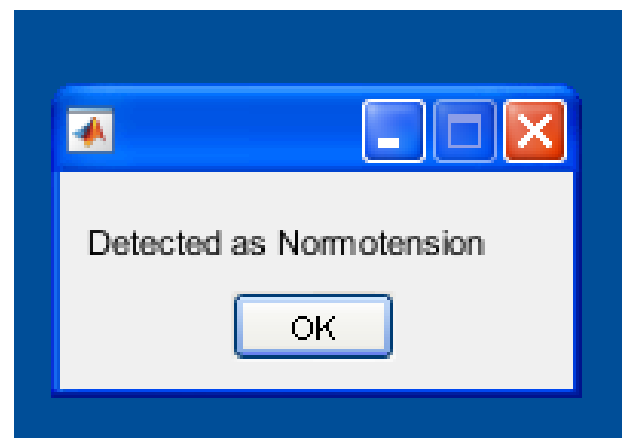

Fig. 12. Detected effect for Test Signal.

The average accuracy is computed given by,

Avg Acc $=\frac{\text { majoirty of class detected }}{\text { Total Number of test conducted }}$

The mean square error is given by,

$M S E=\frac{\sum_{i=1}^{n}\left(x-x^{\prime}\right)^{2}}{n}$

Where $\mathrm{x}$ is the actual signal processing and $\mathrm{x}^{\prime}$ is the denoised coefficient.

PSNR is given by,

PSNR $=\log _{10} \frac{\operatorname{peak}\left(x^{\prime}\right)}{\operatorname{peak}(x)}$

and RMSE is given by,

$R M S E=\sqrt{M S E}$

Observations for the developed approach TLSC is compared with the existing approach of temporal spectral coding (SR) [32], and multi information fusion with neural Network (MIF-NN) [1]. The observation of the developed approaches for denoising for different test samples is presented in Table II.

The test observations developed for different test sample illustrates an enhancement of PSNR by $16 \mathrm{~dB}$ and $5.4 \mathrm{~dB}$ in comparison to SR and MIF-NN method respectively. The time line coding of signal denoising eliminate distortion using a period of observation, wherein a discrete observation generate filtration for a observing coefficients only which has lower filtration performance; more effective denoising results into an accurate signal representation. This result into improve the accuracy of detection. The performance of detection for the developed approaches is listed in Table III.

TABLE II. OBSERVATION FOR THE DEVELOPED APPROACH BASED ON TIME LINE SELECTIVE APPROACH

\begin{tabular}{|l|l|l|l|l|l|}
\hline Test sample & Method & MSE & PSNR(dB) & RMSE & Time(Sec) \\
\hline \multirow{4}{*}{ S1 } & SR & 6.3076 & 34.9088 & 2.5115 & 0.156 \\
\cline { 2 - 6 } & MIF-NN & 1.3557 & 45.4041 & 1.1643 & 0.031 \\
\cline { 2 - 6 } & TLSC & 1.3821 & 50.8703 & 1.1756 & 0.015 \\
\hline \multirow{4}{*}{ S2 } & SR & 6.2818 & 34.4641 & 2.5064 & 0.175 \\
\cline { 2 - 6 } & MIF -NN & 1.3553 & 45.4121 & 1.1642 & 0.029 \\
\cline { 2 - 6 } & TLSC & 1.3821 & 50.8703 & 1.1756 & 0.019 \\
\hline \multirow{5}{*}{ S3 } & SR & 6.2378 & 34.7783 & 2.4976 & 0.168 \\
\cline { 2 - 6 } & MIF -NN & 1.3557 & 45.2999 & 1.1643 & 0.023 \\
\cline { 2 - 6 } & TLSC & 1.3821 & 50.8703 & 1.1756 & 0.011 \\
\hline \multirow{3}{*}{ S4 } & SR & 6.2228 & 34.3509 & 2.4945 & 0.177 \\
\cline { 2 - 6 } & MIF -NN & 1.3560 & 45.4179 & 1.1645 & 0.029 \\
\cline { 2 - 6 } & TLSC & 1.3821 & 50.8703 & 1.1756 & 0.016 \\
\hline
\end{tabular}

TABLE III. ACCURACY OF DETECTION FOR THE DEVELOPED SYSTEMS

\begin{tabular}{|l|l|}
\hline Method of detection & Accuracy $(\%)$ \\
\hline SR & 83.25 \\
\hline MIF-NN & 91.33 \\
\hline TLSC & 94.34 \\
\hline
\end{tabular}

The detection of BP parameters for the estimation accuracy is observed to $94.34 \%$ for the proposed time line processing, wherein the existing approach of SR [32] and MIF-NN [1] has 83.25 and $91.33 \%$ respectively. A more accurate signal denoising results to an improvement of detection of SBP and DBP parameters improve decision accuracy.

"Fig. 13" shows the Observation for MSE for the proposed method. It outperforms the SR method and is almost as good as MIF-NN method.

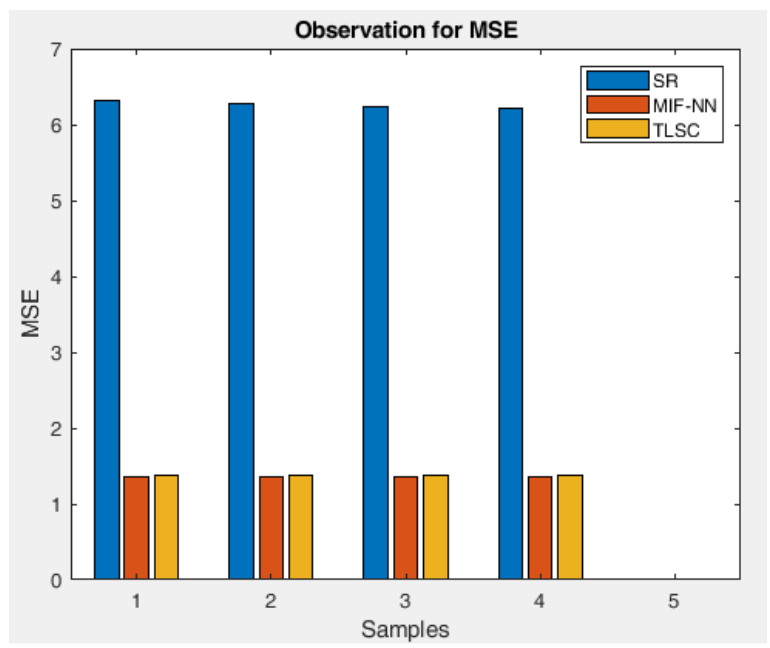

Fig. 13. Observation for MSE Plot. 


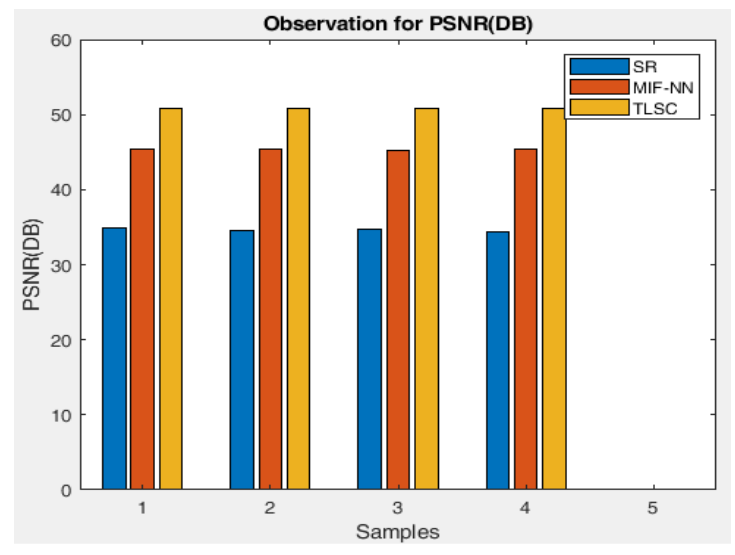

Fig. 14. Observation for PSNR(DB) Plot.

"Fig. 14" shows the Observation for PSNR(DB) plot for the proposed method. The proposed method demonstrates good PSNR values compared with the existing approaches.

"Fig. 15" shows the Observation for RMSE for the proposed method. It outperforms the SR method and is almost as good as MIF-NN method.

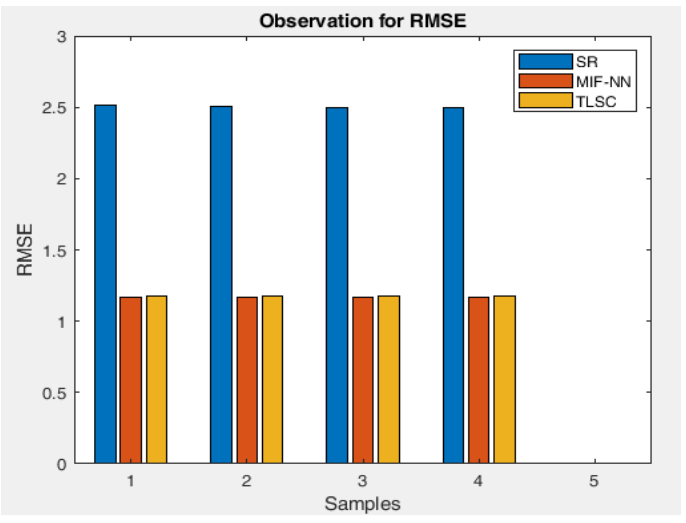

Fig. 15. Observation for RMSE Plot.

\section{DISCUSSION AND FUTURE WORK}

Cuff-Less BP Stratification has gained enormous attention in recent years due to its potential possibility of highly accurate, convenient to use and continuous nature of measurement. However, the accuracy of such measurements largely depends on the quality of captured Vital Bio-Signals used for stratification. Most of the signals are noisy and can lead to problems in BP Stratification. Existing signal denoising and normalization techniques are mostly discarding the wavelet coefficient values above a given threshold, discrete in nature and somewhat lossy. The proposed method in this paper namely Time line correlative spectral processing has demonstrated good results in terms of PSNR values as depicted in the results. It has outperformed the SR and MIF-NN techniques. This will in turn motivate to use this method in practical medical setups for Signal acquisition, filtering and for BP Stratification.

In future we will be using the Time line correlative spectral processing approach for more accurate BP Estimation by processing the PPG Signals by means of optimal feature extraction, selection and application and invention of efficient machine learning algorithm; also to build a temporal dataset of continuous BP measurements and apply big data analytics for more scientific explorations of Cardiac diseases patterns and Hypertension.

\section{VII.CONCLUSION}

A spectral coding approach based on correlative spectral density to implement cuff less Stratification of BP measurement is developed. The coefficients are represented as a continuous time series of measured parameter and the signals is decomposed using sub band coding. Each of the sub bands outlines a variation in frequency domain and is further processed for selection using mean spectral density (MSD). The proposed approach performs a selection for the required bands discarding less informative bands. Selected coefficients are processed for the detection of SBP and DBP parameter in Stratification of BP. The proposed approach shows an improvement of $11 \%$ in average classification accuracy and $16 \mathrm{~dB}$ in PSNR for different tested samples.

\section{ACKNOWLEDGMENT}

We would like to acknowledge Dr. Ramesh Pokale, Physician, Sidhhivinayak Clinic, Nira, Dist: Pune, India for providing the necessary medical support and required information from time to time.

\section{REFERENCES}

[1] Wang, Dingliang, Xuezhi Yang, Xuenan Liu, Shuai Fang, Likun Ma, and Longwei Li. "Photoplethysmography Based Stratification of Blood Pressure Using Multi-Information Fusion Artificial Neural Network." In Proceedings of the IEEE/CVF Conference on Computer Vision and Pattern Recognition Workshops, pp. 276-277. 2020.

[2] Y. Liang, Z. Chen, R. Ward, and M. Elgendi.,"Photoplethysmography and deep learning: Enhancing hypertension risk stratification". Biosensors, 2018.

[3] W. Liu, X. Fang, Q. Chen, Y. Li, and T. Li. ,"Reliability analysis of an integrated device of ECG, PPG and pressure pulse wave for cardiovascular disease". Microelectronics Reliability, 87:183-187, 2018.

[4] I. Pavlidis, J. Dowdall, N. Sun, C. Puri, J. Fei, and M. Garbey. "Interacting with human physiology". Computer Vis. Image Understand, 108(1):150-170, 2017.

[5] L.Tarassenko, A.Hann, and D.Young, "Integrated monitoring and analysis for early warning of patient deterioration", British Journal of Anaesthesia, May 17, 2006.

[6] M. Saeed, M. Villarroel, A. T. Reisner, G. Clifford, L. W. Lehman, G. Moody, T. Heldt, T. H. Kyaw, B. Moody, and R. G. Mark, "Multiparameter intelligent monitoring in intensive care ii: A publicaccess intensive care unit database". Crit. Care Med., 39:952-960, 2011.

[7] Y. Kurylyak, F. Lamonaca, and D. Grimaldi, "A neural network-based method for continuous blood pressure estimation from a PPG signal", IEEE International Instrumentation and Measurement Technology Conference, pages 280-283, 2013.

[8] Alvaro Araujo, Jaime Garcia, Javier Blesa, "Wireless Measurement System for Structural Health Monitoring with High Time Synchronization Accuracy", IEEE Transactions on Instrumentation and Measurement, Vol.61, No.3, March 2012.

[9] M. S. Somayyeh, F. Mohammad, C. Mostafa, H. Mohammad, M. Maryam, and G. Yadollah. ," Blood pressure estimation from appropriate and inappropriate PPG signals using a whole-based method". Biomedical Signal Processing and Control, 47:196-206, 2019.

[10] Alumona T.L, Idigo V.E, and Nnoli, "Remote monitoring of patients health using wireless sensor networks", IPASJ International Journal of Electronics and Communication, vol.2, pp 90-95, Issue 9, September 2014. 
[11] P. Su, X. R. Ding, Y. T. Zhang, J. Liu, F. Miao, and N. Zhao., "Longterm blood pressure prediction with deep recurrent neural networks". In Proceedings of the 2018 IEEE EMBS International Conference on Biomedical and Health Informatics, pages 323-328, 2018.

[12] Abdelhamid.S Mohamed.S and Ajith Abraham, "A review of ambient intelligence assisted health care monitoring",Computer Information Systems and Industrial Management Applications, Vol.5. pp. 741-750, 2013.

[13] Nakamura Masayuki, Nakamura Jiro, Lopez Guillaume., "Collaborative processing of wearable and ambient sensor system for blood pressure monitoring", Sensors Journal, ISSN 1424- 8220, June 2011.

[14] Shahina Begum, Shaibal Barua and Mobyen Uddin Ahmed, "Physiological sensor signals classification for health care using sensor data fusion and case base reasoning", Sensors Journal, 2014.

[15] Chen Xijun, Max Q-H Meng, Ren Hongliang, "Design of sensor node platform for wireless biomedical sensor networks", Proceedings of IEEE, 2005.

[16] A. Visvanathan, A. Sinha, and A. Pal., "Estimation of blood pressure levels from reflective photoplethysmograph using smart phones". IEEE 13th International Conference on Bioinformatics and Bioengineering, 2013.

[17] Tarassenko L, Hann A, Patterson A., "BioSign: multiparameter monitoring for early warning of patient deterioration", Proc 3rd IEEE international Seminar on Medical Applications of Signal Processing, 2005.

[18] L. Wang, W. Zhou, Y. Xing, and X. Zhou. ,"A novel neural network model for blood pressure estimation using photoplethysmography without electrocardiogram, "Journal of Healthcare Engineering", 2018:1-9, 2018

[19] Mane, P.M., Sheela Rani, C.M., High data availability with effective data integrity and user revocation using abe scheme for cloud storage, International Journal of Innovative Technology and Exploring Engineering, 2019.

[20] Mane, S.U., Narsinga Rao, M.R., Large-scale compute-intensive constrained optimization problems: GPGPU-based approach, Advances in Intelligent Systems and Computing,2019.

[21] Meghana, P., Sagar Imambi, S., Sivateja, P., Sairam, K., international Journal of Innovative Technology and Exploring Engineering, Image recognition for automatic number plate surveillance, 2019.
[22] Kumar, S.A., Vidyullatha, P., A comparative analysis of parallel and distributed FSM approaches on large-scale graph data, International Journal of Recent Technology and Engineering,2019.

[23] Sai Meghana, S., Amulya, P., Manisha, A., Rajarajeswari, P., A deep learning approach for brain tumor segmentation using convolution neural network, International Journal of Scientific and Technology Research, 2019.

[24] Sajana, T., Sai Krishna, K., Dinakar, G., Rajdeep, H. Classifying diabetic retinopathy using deep learning architecture, International Journal of Innovative Technology and Exploring Engineering,2019.

[25] Balaraju, J., Prasada Rao, P.V.R.D., Innovative Secure Authentication Interface for Hadoop Cluster Using DNA Cryptography: A Practical Study, Advances in Intelligent Systems and Computing,2020.

[26] Roshini, A., Kiran, K.V.D., Challenges in physiological signal extraction from cognitive radio wireless body area networks for emotion recognition, Advances in Intelligent Systems and Computing,2020.

[27] Sandhya S Waghere , PothuRaju RajaRajeswari, Design and Implementation of System which efficiently retrieve useful data for Detection of Dementia Disease SPRINGER 2021.

[28] Somase, K.P., Imambi, S.S., Develop and implement unsupervised learning through hybrid FFPA clustering in large-scale datasets, Soft Computing,2021.

[29] Anisha, P.R., Vijaya Babu, B., CEBPS: Cluster based effective breast cancer prediction system, International Journal of Recent Technology and Engineering,2019.

[30] Santosh A. Shinde, Dr. P. Raja Rajeswari, "A Novel Hybrid Framework for Cuff-Less Blood Pressure Estimation based On Vital Bio Signals processing using Machine Learning," International Journal of Advanced Trends in Computer Science and Engineering, Vol.9, Issue 2, MarchApril 2020, pp. 1556-1561.

[31] Ms.Bhandare Trupti Vasantrao, Dr. Selvarani Rangasamy, "Review on Heart Disease Diagnosis Using Deep Learning Methods," International Journal of Next-Generation Computing - Special Issue, Vol. 12, No. 2, April 2021, pp. 91-102.

[32] Sundar, Aditya, Vivek Pahwa, Chinmay Das, Mukund Deshmukh, and Neethu Robinson. "A comprehensive assessment of the performance of modern algorithms for enhancement of digital volume pulse signals." International Journal of Pharma Medicine and Biological Sciences 5, No. 1, 2016. 